\title{
BASICS OF LAGRANGIAN FOLIATIONS
}

\author{
I. VAISMAN
}

Abstract

The paper is an exposition of basic known local and global results on Lagrangian foliations such as the theorems of Darboux-Lie, Weinstein, Arnold-Liouville, a global characterization of cotangent bundles, higher order Maslov classes, etc.

The notion of a Lagrangian foliation is a basic one in symplectic geometry and, thereby, in theoretical physics, and still presents many open interesting problems. In the present paper, we want to review some of the basic known results for the benefit of readers who are well aquainted with foliations theory but are less familiar with symplectic geometry, and, also, to present some personal (already published [V2]-[V5]) results.

Our review covers: the Darboux-Lie theorem which gives the local structure of the foliation, and the affine structure of the leaves, Weinstein's theorem of tubular equivalence with a cotangent bundle, the Arnold-Liouville theorem on action-angle coordinates, a global characterization of cotangent bundles, and a few other simple global results, secondary characteristic classes of pairs of Lagrangian foliations, etc.

Details may be found in references such as [LM], [W], [D], [AG], [GS2], [V3], [V4], etc. Everything in the paper is in the $C^{\infty}$-category.

\section{Local Structure Theorems}

Let us recall (see for instance, [LM]) that a symplectic manifold is a manifold $M^{2 n}$ endowed with a closed everywhere nondegenerate 2-form $\omega$, and that a text-book theorem of Darboux then yields a canonical atlas $\left\{\left(q^{i}, p_{i}\right\}\right.$ on $M$ which is characterized by

$$
\omega=d q^{i} \wedge d p_{i} \quad(i=1, \ldots, n) .
$$

This paper has been written while the author was a visitor of the Centre de Recerca Maternatica, Institut d'Estudis Catalans, in the framework of the Semester on Differential Geometry, 1988. The author wishes to express here his gratitude to the CRM, and, particularly, to the professors M. Castellet, J. Girbau, A. Reventós, and M. Nicolau. 
(The Einstein summation convention is used.)

On a symplectic manifold, every function $f$ has an associated Hamiltonian vector field $X_{f}$ characterized by $i\left(X_{f}\right) \omega=d f$, and two functions $f_{1}, f_{2}$ are said to be in involution if their Poisson bracket

$$
\left\{f_{1}, f_{2}\right\}=-\omega\left(X_{f_{1}}, X_{f_{2}}\right)=X_{f_{1}} f_{2}=0 .
$$

All such fields $X_{f}$ are infinitesimal automorphisms of $\omega$ (i.e. $L_{X} \omega=0$ ), and every infinitesimal automorphism is a locally Hamiltonian vector field. Notice also that $\left[X_{f_{1}}, X_{f_{2}}\right]=X_{\left\{f_{1}, f_{2}\right\}}$.

An $n$-dimensional submanifold $L$ of $M$ where $\omega=0$ (or, equivalently, which is locally the set of zeroes of $n$ functions pairwise in involution) is called a Lagrangian submanifold, and a foliation of $M$ whose leaves are Lagrangian submanifolds is called a Lagrangian foliation.

Example 1. $M=T^{*} N^{n}=$ the cotangent bundle of a manifold $N$, with $\omega$ given by (1.1) where $q^{i}$ are local coordinates on $N$, and $p_{i}$ are corresponding covector coordinates, is always a symplectic manifold, and the vertical foliation by fibers $q^{i}=$ const. is a Lagrangian foliation. Particular case: $M=\mathbf{R}^{2 n}=$ $T^{*} R^{n}$. In this case $p_{i}=$ const. also define a horizontal Lagrangian foliation.

Example 2. The torus $M=T^{2 n}$ with cartesian coordinates $\left(q^{i}, p_{i}\right)$ also has the symplectic structure (1.1), and the equations $p_{i}-\alpha^{i} q^{i}=$ const. $(i=$ $1, \ldots, n$; no sum on $i$ ) define a Lagrangian foliation of slopes $\alpha^{i} \in \mathbf{P}$. If all $\alpha^{i}$ are irrational numbers the leaves are dense in $T^{2 n}$.

Example 3. [V4]. The same form (1.1) yields a symplectic structure on the manifold

$$
M_{\lambda}=\left\{\left(\mathbf{R}^{n} \backslash\{0\}\right) \times\left(\mathbb{R}^{n} \backslash\{0\}\right)\right] / K_{\lambda} \approx S^{n-1} \times S^{1} \times S^{n-1} \times \mathbb{R}^{1},
$$

where $q^{i}, p_{i}$ are cartesian coordinates on the two copies of $\mathrm{R}^{n}$, respectively, $K_{\lambda}$ is the group generated by the transfomation $q^{i} \mapsto \lambda q^{i}, p_{i} \mapsto(1 / \lambda) p_{i}<0<$ $\lambda=$ const. $<1)$, and the last diffeomorphism is $(q, p) \mapsto(q /\|q\|, \ln \|q\|, p /\|p\|$, $\ln (\|q\| \cdot\|p\|))$. On $M_{\lambda}$, we have again two Lagrangian foliations respectively given by $q^{i}=$ const., $p_{i}=$ const. Their leaves are diffeomorphic to $S^{n-1} \times R^{1}$. As a matter of fact, $M_{\lambda}$ is $T^{*}\left(S^{n-1} \times S^{1}\right)$ with the zero section removed. If, following a remark by $G$. Hector, we keep the zero section, it will belong to one of the foliations, and the latter will have one compact leaf, while all the other leaves are non compact.

Various other examples are also known (e.g., [V4]). However, it would be interesting to find more examples which would be significant from various viewpoints, particularly from that of foliation theory. The following problem is also open: any Kähler manifold has a symplectic structure given by its Kähler form; find examples (if possible) of Lagrangian foliations on compact Kähler manifolds $M$ which are not complex tori. This problem is difficult for the following reason: the existence of a Lagrangian distribution on $M$ reduces the structure 
group of $T M$ from $U(n)$ to $O(n)$, and, consequently, all the odd Chern classes of $M$ vanish. (See, for instance, $[\mathrm{Bv}]$ for the classification of compact Kähler manifolds with a vanishing first Chern class.) If the strong hypothesis that the foliation be parallel is added, $M$ must be a complex torus ([V3]).

The first basic result about Lagrangian foliations is that, in the same dimension, all such foliations are locally equivalent. This follows from a particular case of a theorem which goes back to $\mathrm{S}$. Lie namely.

Theorem 1.1. (Theorem of Darboux-Lie.) If $\mathcal{L}$ is a Lagrangian foliation of a symplectic manifold $\left(M^{2 n}, \omega\right)$, the latter can be covered by a canonical atlas $\left\{\left(q^{i}, p_{i}\right)\right\}(i=1, \ldots, n)$ such that $\mathcal{L}$ has the local equations $q^{i}=$ const., and $\omega$ is given by (1.1).

Proof: We start by taking local independent first integrals $q^{i}$ of $\mathcal{L}(i=1, \ldots$, $n$ ), in convenient neighbourhoods. Then $\mathcal{L}$ has equations $q^{i}=$ const, and since $\mathcal{L}$ is Lagrangian, the Hamiltonian vector fields $-X_{q^{i}}$ are tangent to $\mathcal{L}$, and $\left[X_{q^{i}}, X_{q^{j}}\right]=X_{\left\{q^{i}, q^{2}\right\}}=0$. Now, it is classical that one can find local coordinates $p_{i}^{i}$ such that $X_{q^{i}}=\partial / \partial p_{i}^{\prime}$, and we see that $\omega$ takes the form

$$
\omega=d q^{i} \wedge d p_{i}^{\prime}+\frac{1}{2} \beta_{i j} d q^{i} \wedge d q^{j} .
$$

Since $\omega$ is closed, the last term of (1.3) is closed as well, whence $\beta_{i j}$ depend locally on $q^{i}$ alone, and the mentioned term equals, say $d\left(\lambda_{i}(q) d q^{2}\right)$. Accordingly, if we take the new local coordinates $p_{i}=p_{i}^{t}-\lambda_{i}(q)$, we are done.

Coordinates such that (1.3), but not necessarilly (1.1), holds are called semicanonical, and it is mainly such coordinates which we shall use in the sequel. An easy computation shows that the transition functions of semicanonical coordinates defined on domains $U_{\alpha}, U_{\beta}$ are locally of the form

$$
q_{\beta}^{i}=q_{\beta}^{i}\left(q_{\alpha}^{j}\right), p_{i}^{\prime \beta}=\sum_{k=1}^{n} \frac{\partial q_{\alpha}^{k}}{\partial q_{\beta}^{i}} p_{k}^{\prime \alpha}+\varphi_{i}^{\alpha \beta}\left(q_{\alpha}^{j}\right),
$$

which proves (e.g., $[\mathbf{W}]$ )

\section{Theorem 1.2 .}

(i) The mapping df $\mapsto-X_{f}$ restricted to germs of projectable functions $f$ defines an isomorphism $V^{*} \mathcal{L} \approx T \mathcal{L}$, where $T \mathcal{L}$ is the tangent bundle, and $V \mathcal{L}$ is the transversal bundle $T M / T \mathcal{L}$ of $\mathcal{L}$.

(ii) The leaves of $\mathcal{L}$ are affine manifolds such that the Hamiltonian fields of projectable functions are the parallel vector fields of the corresponding torsionless fat connection $\stackrel{\circ}{\nabla}$ of the leaves.

It is interesting that (by (i) above) $T \mathcal{L}$ has a canonical structure of a projectable vector bundle, since $\mathcal{V L}$ has such a structure (as for an arbitrary foliation). 
Furthermore a choice of a transversal distribution $T^{\perp} \mathcal{L} \approx \mathcal{V L}$ of $T \mathcal{L}$ yields a projectable structure of $T M$, which is noncanonical, but useful however.

Concerning (ii) of Theorem 1.2, if the restriction of $\stackrel{\circ}{\nabla}$ to each leaf is geodesically complete, the foliation $\mathcal{L}$ is said to be complete. Since compact affine manifolds may be noncomplete, it is difficuit to give completeness results for Lagrangian foliations. For instance, all the leaves of the two Lagrangian foliations of Example 3 are noncomplete (even the compact one if added), since they are affinely covered by $R^{n} \backslash\{0\}$. We quote also the following open problem: assume that the Lagrangian foliation $\mathcal{L}$ has a complete leaf $L_{0}$; which hypotheses would ensure that $L_{0}$ has a saturated neighbourhood consisting of complete leaves?

The ideas which led to (1.3) can also give a more general result namely.

Theorem 1.3. ([W]) Let $\mathcal{L}$ be a Lagrangian foliation of $(M, \omega)$, and let $N$ be an $n$-dimensional embedded submanifold of $M$ which is transversal to the leaves of $\mathcal{L}$. Then, there is an open neighbourhood $U$ of $N$ in $M$ such that $(U, \omega / U, \mathcal{L} / U)$ is equivalent with a neighbourhood $V$ of $N$ seen as the zero section of its cotangent bundle $T^{*} N$, where $T^{*} N$ is endowed with a symplectic form of the semicanonical type (1.3). Moreover if $N$ is a Lagrangian submanifold, $V$ is to be taken with the canonical symplectic structure (1.1).

Proof: Let $U$ be a tubular neighbourhood of $N$ such that $\mathcal{L} / U$ is a simple foliation (every leaf of $\mathcal{L} / U$ has one and only are intersection point with $N$ ). Then the local coordinates $q^{i}$ of $N(i=1, \ldots, n)$ are first integrals of $\mathcal{L} / v$; and they may be used to put $\omega$ in the local form (1.3), while, also, $p_{i}^{\prime}=0$ are the local equations of $N$. This last condition is obtained after performing a translation on the $p_{i}^{\prime}$, if necessary. This may prevent us from making afterwards the other translation indicated at the end of the proof of Theorem 1.1, and, hence, from reaching the canonical form (1.1).

Now, since $p_{i}^{\prime}=0$ have a geometric meaning, the form (1.4) of the transition functions reduces to

$$
q_{\beta}^{i}=q_{\beta}^{i}\left(q_{\alpha}^{j}\right), \quad p_{i}^{\prime \beta}=\sum_{k=1}^{n} \frac{\partial q_{\alpha}^{k}}{\partial q_{\beta}^{i}} p_{k}^{j \alpha},
$$

which are precisely the transition functions of the cotangent bundle $T^{*} N$. This proves our assertion. If $N$ is Lagrangian $p_{i}^{\prime}$ are involutive functions, and (1.3) reduces to (1.1).

A very important local structure theorem of a different kind is the ArnoldLiowville theorem. In order to formulate it let us consider the torus $T^{n}$, and its cotangent bundle $T^{*} T^{n}$ with the canonical symplectic structure (1.1), where $q^{i}$ are cartesian coordinates on $T^{n}$. Then $p_{i}=$ const. is a Lagrangian foliation of $T^{*} T^{n}$ which is transversal to the foliation by fibers, and whose leaves are the orbits of the natural free action of $T^{n}$ on $T^{*} T^{n}$. We call this the horizontal foliation of $T^{*} T^{n}$. 
Theorem 1.4. (Liouville, Arnold) Let $\mathcal{L}$ be a Lagrangian foliation of $(M, \omega)$, and $L_{0}$ be a leaf of $\mathcal{L}$ which is compact and has a trivial holonomy. Then $L_{0}$ has an open $\mathcal{L}$-saturated neighbourhood $U$ in $M$ such that $(U, \omega / U, \mathcal{L} / U)$ is equivalent to the horizontal foliation of $T^{*} T^{n}$ in a neighbourhood of the zero section, the latter being the image of $L_{0}$ by the mentioned equivalence. Particularly, $\mathcal{L} / v$ consists of the orbits of a free action of $T^{n}$ by symplectic equivalences of $U$.

Proof: See for instance [D]. As a consequence of the well known local stability theorem, under the given hypotheses $L_{0}$ has an open saturated neighbourhood $U$ which is diffeomorphic to $V \times L_{0}$, for some open disk $V$ of $\mathbf{R}^{n}$ centered at the origin, and such that the leaves of $\mathcal{L} / U$ are the fibers of the projection onto $V, L_{0}$ corresponding to the origin. Hence $\mathcal{L} / U$ may be seen as the foliation defined by equations $f_{i}=$ const., where $f_{i}$ (the cartesian coordinates in $V$ ) are functions on $U$, which are functionally independent and pairwise in involution. The Hamiltonian vector fields $X_{f}$ are globally defined and everywhere independent on the leaves of $\mathcal{L} / U$, and, since the leaves are compact, $X_{f_{i}}$ are complete vector fields. They also commute $\left(\left\{f_{i}, f_{j}\right\}=0 \Rightarrow\left[X_{f_{i}}, X_{f_{j}}\right]=X_{\left\{f_{i}, f_{j}\right\}}=0\right)$, and, hence their flows define symplectomorphisms of $U$ which yield a transitive and locally free action of $\mathbb{R}^{n}$ on the leaves of $\mathcal{L} / U$ with the discrete isotropy subgroups $G_{x_{0}} \subset \mathbb{R}^{n}$, where $x_{0}$ runs on a cross section of $U$ over $V$.

Accordingly, the leaves of $\mathcal{L} / U$ are tori $\mathrm{R}^{n} / G_{x_{0}}$, and have angular coordinates $\varphi^{\prime i} \in[0,2 \pi]$ such that

$$
\frac{\partial}{\partial \varphi^{\prime i}}=\sum_{j=1}^{n} \xi_{i}^{j} X_{f_{j}},
$$

where $\xi_{i}^{j}=\xi_{i}^{j}\left(f_{k}\right)$, det $\left(\xi_{i}^{j}\right) \neq 0$, and the flows of $\partial / \partial \varphi^{\prime i}$ are symplectomorphisms of $U$. Since our functions are defined locally, these properties imply that a local change of coordinates $s_{i}=s_{i}\left(f_{k}\right)$ can be done such that $X_{s_{i}}=-\left(\partial / \partial \varphi^{i}\right)$.

The local coordinates $\left(\varphi^{\prime 2}, s_{i}\right)$ satisfy

$$
\left\{s_{i}, s_{j}\right\}=0, \quad\left\{\varphi^{\prime j}, s_{i}\right\}=\delta_{i}^{j} .
$$

Hence, they are semicanonical coordinates for $\omega / U$, and, like in the proof of Theorem 1.1, translations

$$
\varphi^{i}=\varphi^{\prime i}+\psi^{i}(s)
$$

can be found such that $\left(s_{i}, \varphi^{j}\right)$ be canonical coordinates of $\omega / U$ (i.e., besides (1.7) also $\left\{\varphi^{i}, \varphi^{j}\right\}=0$ holds, and $\omega / U=d \varphi^{i} \wedge d s_{i}$.

Clearly $\varphi^{i}$ are also angular coordinates on the tori-leaves of $\mathcal{L} / U$, while (in view of a mechanical interpretation) $s_{i}$ are called action coordinates. If, with 
reference to the identification $L_{0} \approx T^{n}$, we define the mapping $U \rightarrow T^{*} T^{n}$ given by $q^{i}=\varphi^{i}, p_{i}=s_{i}$, we get exactly the equivalence announced by Theorem 1.4 .

The existence of action angle coordinates is very important for the integration of Hamiltonian dynamical systems (e.g., [LM]).

A globalization of Theorem 1.4 for locally trivial fbrer bundles with Lagrangian fibre was given by Duistermaat [D]. Generalizations from $T^{n}$ to noncommutative Lie groups $G$ where given by Dazord and Delzant [DD], [Dz].

\section{Simple Global Results}

In view of the local results of Section 1, it is natural to ask for conditions of global equivalence of a Lagrangian foliation with the fiber foliation of a cotangent bundle. In order to formulate an answer, we define first a certain cohomological obstruction [V4].

Let $\left\{U_{\alpha} ;\left(q_{\alpha}^{i}, p_{i}^{\alpha}\right)\right\}_{\alpha \in A}$ be an atlas of semicanonical coordinates of the symplectic manifold $(M, \omega)$ with respect to its Lagrangian foliation $\mathcal{L}$. Hence, formulas (1.3) and (1.4) hold good. Then, we define the local Euler vector fields

$$
E_{\alpha}=\sum_{i=1}^{n} p_{i}^{\alpha} \frac{\partial}{\partial p_{i}^{\alpha}}
$$

on $U_{\alpha}$, and notice that (1.4) implies

$$
E_{\beta}-E_{\alpha}=\sum_{i=1}^{n} \varphi_{i}^{\alpha \beta}\left(q_{\alpha}\right) \frac{\partial}{\partial p_{i}^{\beta}} .
$$

Accordingly, $\left\{E_{\beta}-E_{\alpha}\right\}$ define a 1-cocycle of $M$ with coefficients in the sheaf $T \mathcal{L}$ of germs of projectable sections of the tangent bundle of $\mathcal{L}$. (Remember that Theorem 1.2. (i) gave us a projectable structure on $T \mathcal{L}$ ), and we get a corresponding cohomology class

$$
\mathcal{E}(\mathcal{L}) \in H^{1}(M, \underset{T}{T} \mathcal{L})
$$

which we call the Euler obstruction of $\mathcal{C}$.

Theorem 2.1. [V4] The triple $(M, \omega, \mathcal{L})$ is globally equivalent to a cotangent bundle $T^{*} N$ endowed with a semicanonical symplectic form iff: i) $\mathcal{L}$ is complete; ii) the leaves of $\mathcal{L}$ are simply connected, iii) $\mathcal{E}(\mathcal{L})=0$.

Proof: i), ii), and iii) are obviously necessary conditions for the fiber foliation of a cotangent bundle. Particularly, iii) follows since, in this case, $\varphi_{i}^{\alpha \beta}=0$ in (1.4). 
Conversely, by $(2.2), \mathcal{E}(\mathcal{L})=0$ means

$$
\varphi_{i}^{\alpha \beta}\left(q_{\alpha}\right) \frac{\partial}{\partial p_{i}^{\beta}}=\psi_{i}^{\beta}\left(q_{\beta}\right) \frac{\partial}{\partial p_{i}^{\beta}}-\psi_{i}^{\alpha}\left(q_{\alpha}\right) \frac{\partial}{\partial p_{i}^{\alpha}},
$$

where we have summation on $i$, and $\sum_{i} \psi_{i}^{\alpha}\left(q_{\alpha}\right)\left(\partial / \partial p_{i}^{\alpha}\right)$ define a 0 -cochain in $\stackrel{T}{=}$. Then

$$
\tilde{q}_{\alpha}^{i}=q_{\alpha}^{i}, \quad \tilde{p}_{\alpha}^{i}=p_{\alpha}^{i}-\psi_{i}^{\alpha}\left(q_{\alpha}\right)
$$

give a semi-canonical atlas with vanishing terms $\varphi_{i}^{\alpha \beta}$ in (1.4). For this new atlas, (2.1) yields a global Euler vector field $E$.

Hypotheses i), ii) show that the leaves of $\mathcal{L}$ are affinely equal to $\mathbf{R}^{n}$. Let $L$ be one such leaf, $x_{0} \in L \cap U_{\alpha}$, and $\xi_{i}^{L}$ be the global affine coordinates defined on $L$ by the exponential mapping of the leafwise fat connection $\stackrel{\circ}{\nabla}$ (Theorem 1.2 , (ii)) at $x_{0}$. It follows easily that $\xi_{i}^{L}=\tilde{p}_{i}^{\alpha}-\tilde{p}_{i}^{\alpha}\left(x_{0}\right)$ on $L \cap U_{\alpha}$, and

$$
E / L=\sum_{i=1}^{n}\left(\xi_{i}^{L}+\tilde{p}_{i}^{\alpha}\left(x_{0}\right)\right) \frac{\partial}{\partial \xi_{L}^{i}} .
$$

Hence $E$ has one and only one vanishing point $z(L)$ on each leaf $L$, and if $z(L)$ belongs to some $U_{\sigma}$, the locus $N$ of $z(L), L \in \mathcal{L}$, has equations $\tilde{p}_{i}^{\sigma}=0$ in $U_{\sigma}$.

It follows that $N$ is a differentiable manifold with the local coordinates $\widetilde{q}_{i}^{\sigma}$, and the local coordinates $\left(\tilde{q}_{\sigma}^{2}, \xi_{i}^{L}\right)$ yield the equivalence of $(M, \omega, \mathcal{L})$ with the fiber foliation of $T^{*} N$. Notice that the canonical form is obtained on $T^{*} N$ by the construction above iff $N$ is a Lagrangian submanifold of $M$.

The previous result may be completed by

Theorem 2.2. Let $\left(M_{a}, \omega_{a}, \mathcal{L}_{a}\right)(a=1,2)$ be two Lagrangianly foliated manifolds which satisfy the conditions of Theorem 2.1. Then, they are equivalent iff there exists a foliation equivalence $F:\left(M_{1}, \mathcal{L}_{1}\right) \rightarrow\left(M_{2}, \mathcal{L}_{2}\right)$ such that $\omega_{1}-F^{*} \omega_{2}$ is a projectably exact form.

Proof: If the equivalence requested exists we may use it as $F$, and then $\omega_{1}-F^{*} \omega_{2}=0$.

Conversely, if $F$ exists, the construction of Theorem 2.1 yields two differentiably equivalent cotangent bundles $T^{*} N_{a}(a=1,2)$ with local coordinates $\left(\tilde{q}_{a}^{i}, \xi_{i}^{a}\right)$, and with two symplectic forms

$$
\omega_{a}=\widetilde{d q_{a}^{i}} \wedge d \xi_{i}^{a}+\beta_{a}
$$

where $\beta_{a}$ are closed projectable 2 -forms.

The equations of $F$ must have the local form

$$
\widetilde{q}_{2}^{i}=\tilde{q}_{1}^{i}, \quad \xi_{i}^{2}=\xi_{1}^{2}\left(\tilde{q}_{1}^{j}, \xi_{j}^{1}\right),
$$


such that $\omega_{2}-F^{*} \omega_{1}=d \lambda$, where $\lambda=\lambda_{i}\left(\tilde{q}_{1}^{j}\right) d q_{1}^{i}$. The lattest condition immediately implies that the second equation (2.5) must be of the form $\xi_{1}^{2}=$ $=\xi_{i}^{1}+\alpha_{i}\left(q_{1}^{j}\right)$, and that the mapping $S: M_{1} \rightarrow M_{2}$ defined by

$$
\tilde{q}_{2}^{i}=\tilde{q}_{1}^{i}, \xi_{i}^{2}=\xi_{i}^{1}+\alpha_{i}-\lambda_{i}
$$

(which has a global meaning) is an equivalence $\left(M_{1}, \omega_{1}, \mathcal{L}_{1}\right) \approx\left(M_{2}, \omega_{2}, \mathcal{L}_{2}\right)$.

Remarks.

1) For simple Lagrangian foliations $\mathcal{L}$ it suffices to assume only hypotheses i) and ii) in Theorem 2.1, and the results of Theorems 2.1 and 2.2 are contained in $[\mathbf{A G}]$.

2) Example 3 of Section 1 gives a Lagrangian foliation $\mathcal{L}$ with non simply connected leaves, and $\mathcal{E}(\mathcal{L})=0$.

3) $\mathcal{E}(\mathcal{L})=0$ is equivalent with the existence of a 1 -form $\lambda=p_{i} d q^{i}$, where $d q^{i}=0$ defines $\mathcal{L}$, such that $\omega-d \lambda$ is projectable. Indeed, then $\left\{\left(q^{i}, p_{i}\right)\right\}$ is a semicanonical atlas with vanishing terms $\varphi_{i}^{\alpha \beta}$ in (1.4). From this, it follows that $\mathcal{E}(\mathcal{L})$ never vanishes on a compact manifold $M$ since $\omega^{n}=(d \lambda)^{n}$.

4) A similar cohomology class like $\mathcal{E}(\mathcal{L})$ appeared in the theory of affine manifolds, namely the radiance obstruction [GH].

Another interesting question is that of the global rigidity of a Lagrangian foliation. To be more precise, the existence of the local canonical coordinates of Theorem 1.1 for a triple $(M, \omega, \mathcal{L})$ means that $M$ is endowed with some pseudogroup structure, and we should study the deformations of this pseudogroup structure (e.g., [GS1]). Following is one simple result in this direction.

Theorem 2.3. Let $N^{n}$ be a manifold such that $H^{1}(N, \mathbf{R})=H^{2}(N, \mathbf{R})=0$. Then the fiber foliation of $T^{*} N$ is infinitesimally rigid as a Lagrangian foliation.

Proof: Infinitesimal rigidity of our pseudogroup structure holds if $H^{1}\left(T^{*} N, \Xi\right)=0$, where $\Xi$ is the sheaf of germs of infinitesimal automorphisms of the structure [GS1]. For any $(M, \omega, \mathcal{L})$, a germ of $\Xi$ is represented by a vector field which preserves $\mathcal{L}$ and $\omega$, and this means

$$
X=\xi^{i}(q) \frac{\partial}{\partial q^{i}}+\eta_{i}(p, q) \frac{\partial}{\partial p_{i}},
$$

where $\left(q^{i}, p_{i}\right)$ are canonical coordinates, and

$$
\frac{\partial \eta_{i}}{\partial p_{j}}=-\frac{\partial \xi^{j}}{\partial q^{i}}, \frac{\partial \eta_{i}}{\partial q^{j}}=\frac{\partial \eta_{j}}{\partial q^{i}}
$$

For $X$ of (2.7), if we write down $i(X) \omega$ in canonical coordinates, we see that $i(X) \omega=d f$ for a germ of a certain function $f$ of the form $\alpha^{i}(q) p_{i}+\beta(q)$, and if we denote by $\mathcal{A}$ the sheaf of germs of such affine functions, we get an isomorphism $\Xi \approx d \mathcal{A}$, and an exact sequence of sheaves

$$
0 \longrightarrow \mathbf{R} \longrightarrow \mathcal{A} \stackrel{d}{\longrightarrow}(d \mathcal{A} \approx \Xi) \longrightarrow 0 .
$$


Accordingly, under the hypotheses of Theorem 2.3 , we get $H^{1}\left(T^{*} N, \Xi\right)=$ $H^{1}\left(T^{*} N, \mathcal{A}\right)$.

But the cohomology of $\mathcal{A}$ can be computed. Indeed, for any $(M, \mathcal{L}, \omega)$, if $\Phi$ denotes the sheaf of germs of projectable functions, $\mathcal{A}$ is a locally free $\Phi-$ module sheaf generated by $\left\{p_{i}, 1\right\}$. Hence $\mathcal{A}$ is the sheaf of germs of projectable sections in a certain projectable vector bundle, and we have [VI]

$$
H^{k}(M, \mathcal{A})=\frac{\operatorname{ker}\left\{d_{f}: \Gamma\left(\mathcal{A} \otimes_{\Phi} \Delta^{0 k} \mathcal{L}\right) \rightarrow \Gamma\left(\mathcal{A} \otimes_{\Phi} \Delta^{0, k+1} \mathcal{L}\right)\right\}}{\operatorname{im}\left\{d_{f}: \Gamma\left(\mathcal{A} \otimes_{\Phi} \Delta^{0, k-1} \mathcal{L}\right) \rightarrow \Gamma\left(\mathcal{A} \otimes_{\Phi} \Delta^{0 k} \mathcal{L}\right)\right\}}
$$

where $d_{f}$ is the exterior differential along the leaves, $\Gamma$ denotes global section spaces, and $\Lambda^{0 k} \mathcal{L}$ is the sheaf of germs of diferential $k$-forms containing only differentials of transversal coordinates of $\mathcal{L}$.

In our case $M=T^{*} N$, and $H^{*}\left(T^{*} N, \mathcal{A}\right)$ is the limit of a spectral sequence with $E_{2}^{h k}=H^{h}\left(N, \mathcal{H}^{k}\left(" F^{\prime \prime}, \mathcal{A}\right)\right.$ ), where $\mathcal{H}^{k}\left(" F^{\prime \prime}, \mathcal{A}\right)$ is the sheaf defined by $U C$ $N \mapsto H^{k}\left(\pi^{-1}(U), \mathcal{A}\right)$ ( $U$ is open, and $\pi: T^{*} N \rightarrow N$ is the natural projection). From $(2.10)$, and since the fibers of $\pi$ are contractible, we get $\mathcal{H}^{k}\left(" F^{\prime \prime}, \mathcal{A}\right)=0$ for $k \geq 1, H^{0}\left(\pi^{-1}(U), \mathcal{A}\right)=\left[C^{\infty}(U)\right]^{n+1}$. This implies $E_{2}^{h k}=0$ for $k \geq 1$, and for $k=0, h \geq 1$. Therefore $H^{k}\left(T^{*} N, \mathcal{A}\right)=0$ for $k \geq 1$, and we are done.

Another important global question is that of the existence of a transversal projectable connection for a Lagrangian foliation $\mathcal{L}$. It turns out that this question is related to the interesting notion of an affine transversal distribution [M1], which is defined as a transversal distribution $\tau \mathcal{L}$ of $\mathcal{L}$ such that the natural process of lifting paths from a transversal submanifold of $\mathcal{L}$ to paths tangent to $\tau \mathcal{L}$ yields affine mappings between the leaves of $\mathcal{L}$. Such a distribution has local equations of the form

$$
\theta_{i}=d p_{i}+t_{i j}(p, q) d q^{j}=0
$$

where $\left(q^{i}, p_{i}\right)$ are sernicanonical coordinates and $t_{i j}$ are affine functions. The existence of an affine transversal distribution is characterized by the vanishing of a certain cohomological obstruction which we shall not describe here [M2], [V4]. But we give

Theorem 2.4. [V4] If $\mathcal{L}$ has an affine transversal distribution, the transversal bundle $\mathcal{V L}$ has a projectable connection. The converse also holds if $\mathcal{E}(\mathcal{L})=0$

Proof: Since $\mathcal{V} \mathcal{L} \approx T^{*} \mathcal{L}$ (theorem $\left.1.1, \mathrm{i}\right)$ ) it suffices to prove the results of Theorem 2.4 for the tangent bundle $T \mathcal{L}$ instead of $V \mathcal{L}$. On $T \mathcal{L}$, for any transversal distribution $\tau \mathcal{L}$, we may extend the flat partial connection $\stackrel{\circ}{\nabla}$ along the leaves (Theorem 1.2, ii)) to a full connection by adding to it

$$
\stackrel{\circ}{\nabla}_{X} Y=\operatorname{pr}_{T}[X, Y], \quad \forall X \in \tau \mathcal{L}, \quad Y \in T \mathcal{L} .
$$


If $\stackrel{\circ}{R}$ denotes the curvature operator of this connection $\stackrel{\circ}{\nabla}$, we obviously have $\stackrel{\circ}{R}\left(Y_{1}, Y_{2}\right) Y=0$ for all $Y_{1}, Y_{2}, Y \in T \mathcal{L}$, and the connection will be projectable iff $\stackrel{\circ}{R}\left(X, Y_{1}\right) Y=0$ for $X \in \tau \mathcal{L}, Y_{1}, Y \in T \mathcal{L}[\mathrm{M} 1]$.

By definition, $\tau \mathcal{L}$ is affine iff for every foliation preserving infinitesimal transformation $X \in \tau \mathcal{L}$, and every parallel field $Y \in T \mathcal{L},[X, Y]$ is again a parallel field. In the computation of $\stackrel{\circ}{R}\left(X, Y_{1}\right) Y$, we may always assume that $X$ is leaf preserving and $Y_{1}, Y$ are parallel (since the result depends only on the point-values of these vector fields). By doing this, we get easily $\stackrel{\circ}{R}\left(X, Y_{1}\right) Y=0$.

Conversely, assume that $T \mathcal{L}$ has a projectable connection, and $\mathcal{E}(\mathcal{L})=$ $=0$. Then a germ of $M$ may be seen as a germ of the cotangent bundle of a transversal submanifold of $\mathcal{L}$, and the projectable connection of $T \mathcal{L}$ induces a connection $\nabla$ in this cotangent bundle. Locally (and if $\left(q^{i}, p_{i}\right)$ are again the canonical coordinates of a cotangent bundle), $\nabla$ may be written as

$$
\nabla_{\left(\partial / \partial q^{i}\right)}\left(d q^{j}\right)=\sum_{k} \Gamma_{j i}^{k}(q)\left(d q^{k}\right)
$$

(Here $\left(d q^{j}\right)$ is seen as the basis of the fibers of the cotangent bundle.) The tangent vector of the paths of $\nabla$-parallelism satisfy

$$
d p_{k}+\sum_{i, j} \Gamma_{i j}^{k}(q) p_{i} d q^{j}=0
$$

and we see that they define an affine transversal distribution $\tau \mathcal{L}$. (See details in $[\mathrm{V} 4])$.

\section{Pairs of Lagrangian Foliations}

This is again an important configuration for physical interpretations (particularly for quantum physics). Such pairs appear in $R^{2 n}$. Other examples are offered by Examples 2,3 of Section I.

A geometrically simple but rarely appearing case is that of a pair $\left(\mathcal{L}_{1}, \mathcal{L}_{2}\right)$ of everywhere transversal Lagrangian foliations on a symplectic manifold $(M, \omega)$. Such a system $\left(M, \omega, \mathcal{L}_{1}, \mathcal{L}_{2}\right)$ can be called a bilagrangian manifold.

Theorem 3.1. A bilagrangian manifold has a canonical torsionless symplectic connection $\nabla$ which preserves $\mathcal{L}_{1}, \mathcal{L}_{2} . \nabla$ has zero curvature iff either i) the manifold is locally equivalent to $\mathrm{R}^{2 n}$ with the horizontal and vertical foliations, or ii) $T \mathcal{L}_{2}$ is an affine transversal distribution of $\mathcal{L}_{1}$.

Proof: (E.g., [V2], [V4]) We shall denote by indices $a=1,2$ the components along $\mathcal{L}_{1}, \mathcal{L}_{2}$, and define $\nabla$ as a sum of connections. Start by

$$
\nabla_{X_{1}} Y_{2}=\left[X_{1}, Y_{2}\right]_{2}, \nabla_{X_{2}} Y_{1}=\left\{X_{2}, Y_{1}\right]_{1}
$$


Then ask $\nabla \omega=0$ which yields

$$
\begin{aligned}
& \omega\left(\nabla_{X_{1}} Y_{1}, Z_{1}\right)=0, \omega\left(\nabla_{X_{2}} Y_{2}, Z_{2}\right)=0 \\
& \omega\left(\nabla_{X_{1}} Y_{1}, Z_{2}\right)=X_{1}\left(\omega\left(Y_{1}, Z_{2}\right)\right)-\omega\left(Y_{1},\left[X_{1}, Z_{2}\right]_{2}\right), \\
& \omega\left(\nabla_{X_{2}} Y_{2}, Z_{1}\right)=X_{2}\left(\omega\left(Y_{2}, Z_{1}\right)\right)-\omega\left(Y_{2},\left[X_{2}, Z_{1}\right]_{1}\right) .
\end{aligned}
$$

$\nabla$ is thereby determined, and the computation of its torsion gives 0 in view of the integrability of $\mathcal{L}_{1}, \mathcal{L}_{2}$.

Now, $\nabla$ can be seen as a connection on the principal bundle of frames $\left(E_{i}, E_{i+n}\right)(i=1, \ldots, n)$ which put $\omega$ in the canonical form (i.e., $\omega\left(E_{i}, E_{j+n}\right)=$ $\left.=-\omega\left(E_{i+n}, E_{j}\right)=\delta_{i j}\right)$, and $E_{i} \in T \mathcal{L}_{1}, E_{i+n} \in T \mathcal{L}_{2}$. It follows that $\nabla$ has zero curvature iff there are local parallel frame fields of this type. Moreover, since $\nabla$ has no torsion the corresponding parallel vector fields $E_{i}, E_{j+n}$ commute, and we may define $E_{i}=\partial / \partial q^{i}, E_{j+n}=\partial / \partial p_{j}$. The local coordinates $\left(q^{i}, p_{j}\right)$ show the local equivalence with $\mathrm{R}^{2 n}$.

Finally, in $\mathbb{A}^{2 n}, T \mathcal{L}_{2}$ is of course affine for $\mathcal{L}_{1}$. To get the converse result, let $\left(q^{2}, p_{2}\right)$ be canonical coordinates for $\mathcal{L}_{1}$ (particularly, $d q^{i}=0$ defines $\mathcal{L}_{1}$ ), and let then $\mathcal{L}_{2}$ be spanned by $Q_{i}=\left(\partial / \partial q^{i}\right)-t_{i}^{j}\left(\partial / \partial p_{j}\right)$. Then (3.1), (3.2) yield the following local equations of $\nabla$

$$
\begin{aligned}
& \nabla_{\partial / \partial_{p_{i}}}\left(\partial / \partial p_{j}\right)=0, \nabla_{Q_{i}}\left(\partial / \partial p_{j}\right)=\sum_{k=1}^{n} \frac{\partial t_{i}^{k}}{\partial p_{j}} \frac{\partial}{\partial p_{k}}, \\
& \nabla_{\partial / \partial_{p_{i}}} Q_{3}=0, \quad \nabla_{Q_{i}} Q_{j}=-\sum_{k=1}^{n} \frac{\partial t_{j}^{j}}{\partial p_{k}} Q_{k} .
\end{aligned}
$$

The integrability condition of $\mathcal{L}_{2}$ is

$$
\tau_{i j k}=\frac{\partial t_{i}^{h}}{\partial x^{j}}-\frac{\partial t_{j}^{h}}{\partial x^{i}}+\sum_{s=1}^{n} t_{i}^{s} \frac{\partial t_{j}^{h}}{\partial p_{s}}-\sum_{s=1}^{n} t_{j}^{s} \frac{\partial t_{i}^{h}}{\partial p_{s}}=0,
$$

and using this, the only perhaps nonvanishing parts of the curvature of $\nabla$ will be

$$
\begin{aligned}
& \Omega\left(\frac{\partial}{\partial p_{i}}, Q_{j}\right) \frac{\partial}{\partial p_{k}}=\sum_{h=1}^{n} \frac{\partial^{2} i_{j}^{h}}{\partial p_{k} \partial p_{i}} \frac{\partial}{\partial p_{h}} \\
& \Omega\left(\frac{\partial}{\partial p_{i}}, Q_{j}\right) Q_{k}=-\sum_{h=1}^{n} \frac{\partial^{2} t_{j}^{k}}{\partial p_{i} \partial p_{h}} Q_{h} .
\end{aligned}
$$

Hence $\nabla=0$ iff $t_{i}^{j}$ are affine functions.

(See [V4] for other results about the curvature of bilagrangian manifolds.) More often, pairs of Lagrangian manifolds will have a certain nontransversality set $S \subset M$. Nontransversality leads to the existence of some interesting secondary characteristic classes which, in fact, appear in the more general situation of a pair of Lagrangian subbundles $L_{2}, L_{2}$ of a symplectic vector bundle 
$\pi: E \rightarrow M$ i.e, a bundle with $2 n$-dimensional fibres, and endowed with an everywhere nondegenerate cross-section $\omega$ of $\Lambda^{2} E$. A Lagrangian subbundle is defined in the same way as a Lagrangian submanifold. The simplest such class in the famous Maslov class of the asymptotic analysis of the partial differential equations of quantum physics [GS2]. We give a short description of these characteristic classes, while refering the interested reader to [V3] for details.

Since the unitary group $U(n)$ is a compact maximal subgroup of the symplectic group $S p(n, \mathrm{P})$, the structure group of a symplectic vector bundle $E \rightarrow M$ is reducible to $U(n)$, and, in fact, there is a homotopy class of such reductions. Let us fix one such reduction, and let $J$ be the corresponding complex structure tensor of $E$. Let us look at the principal bundle $\mathcal{U}(E, J) \rightarrow M$ of the unitary frames $\left(e_{i}, J e_{i}\right)(i=1, \ldots, n)$, or in the complex form

$$
\epsilon_{i}=\frac{1}{\sqrt{2}}\left(e_{i}-\sqrt{-1} J e_{i}\right)
$$

of the reduced structure. Then a connection $\theta$ on $\mathcal{U}(E, J)$ has local equations

$$
\nabla \epsilon_{i}=\theta_{i}^{j} \epsilon_{j}
$$

and curvature forms

$$
\Theta_{i}^{j}=d \theta_{i}^{j}-\theta_{i}^{h} \wedge \theta_{h}^{j}
$$

where $\theta$ and $\theta$ take values in the unitary Lie algebra $u(n)$.

Accordingly, we get the real Chern classes $c_{k}(E)(k=1, \ldots, n)$ which do not depend on the choice of $J$ (since two such choices are homotopically related), and they are represented by the differential forms

$$
\Delta(\theta) c_{k}=\frac{(-1)^{k}}{(2 \pi \sqrt{-1})^{k} k !} \delta_{i_{1} \ldots i_{k}}^{j_{1} \ldots j_{k}} \Theta_{j_{1}}^{i_{1}} \wedge \cdots \wedge \Theta_{j_{k}}^{i_{k}}
$$

where $c_{k}$ are the Chern polynomials

$$
c_{k}(A)=\left(\frac{-1}{2 \pi \sqrt{-1}}\right)^{k} \operatorname{tr} \wedge^{k} A \quad(A \in u(n))
$$

and $\triangle(\theta)$ is the classical Chern-Weil homomorphism.

A Lagrangian subbundle $L$ of $E$ is equivalent to a further reduction of the structure group of $E$ from $U(n)$ to $O(n)$. The corresponding principal subbundle of frames $\mathcal{U}(E, J, L)$ consists of unitary frames (3.4) where $e_{i} \in L$, and corresponding connections (3.5) will have antisymmetric matrices $\theta, \Theta$. Such connections will be called $L$-orthogonal unitary connections, if seen on $\mathcal{U}(E, J)$, and for then we obviously have $\Delta(\theta) c_{2 h-1}=0(h=1,2, \ldots)$. 
Now, let $L_{a}(a=1,2)$ be two Lagrangian subbundles of $E$, and $\theta_{a}$ be $L_{a}-$ orthogonal unitary connections. Let $\Delta\left(\theta_{1}, \theta_{2}\right)$ denote the comparison mapping defined by the two connections (e.g., [V3]). The classical formula

$$
\triangle\left(\theta_{2}\right) c_{k}-\triangle\left(\theta_{1}\right) c_{k}=d \Delta\left(\theta_{1}, \theta_{2}\right) c_{k}
$$

shows that $\Delta\left(\theta_{1}, \theta_{2}\right) c_{2 k-1}$ are closed forms, and, therefore, define cohomology classes

$$
\mu_{h}\left(E, L_{1}, L_{2}\right)=\left[\triangle\left(\theta_{1}, \theta_{2}\right) c_{2 h-1}\right] \in H^{4 h-3}(M, \mathbf{R}) .
$$

We call $\mu_{h}$ the $h^{t h}$ Maslov class since $\mu_{1}$ coincides (up to a constant factor) with the original Maslov class.

The explicitation of (3.10) is as follows. Express both connections $\theta_{a}$ by using some common local unitary bases $\left(\epsilon_{i}\right)$, and let $\left(\gamma_{a i}^{j}\right)$ be the corresponding connection matrices. (They take values in $u(n)$ and not in $l(n)=$ the orthogonal Lie algebra, in general.) Put

$$
\alpha_{i}^{j}=\gamma_{2 i}^{j}-\gamma_{1 i}^{j}, \quad \gamma_{t}=\gamma_{1}+t \alpha, \quad 0 \leq t \leq 1,
$$

and let $\Theta_{t}$ be the curvature matrix of $\gamma_{t}$. Then

$$
\begin{aligned}
& \triangle\left(\theta_{0}, \theta_{1}\right) c_{2 h-1}=(-1)^{h+1} \frac{\sqrt{-1}}{(2 \pi)^{2 h-1}(2 h-2) !} . \\
& \int_{0}^{1}\left\{\delta_{i_{1} \ldots i_{2 h-1}}^{j_{1} \ldots j_{2 h-1}} \alpha_{j_{1}}^{i_{1}} \wedge \Theta_{t j_{2}}^{i_{2}} \wedge \cdots \wedge \Theta_{t_{j h-1}}^{i_{2 h-1}}\right\} d t .
\end{aligned}
$$

The general construction of secondary characteristic classes, analogous to the one in foliation theory (e.g., [L1]) amounts to the following. Take the differential graded algebra

$$
\begin{aligned}
& W L_{n}=\mathbb{R}\left[c_{2}^{1}, c_{4}^{1}, \ldots, c_{2[n / 2]}^{1}\right] \otimes \\
& \otimes \mathbb{R}\left[c_{2}^{2}, c_{4}^{2}, \ldots, c_{2[n / 2]}^{2}\right] \otimes \\
& \otimes \wedge\left(u_{1}, u_{2}, \ldots, u_{n}\right),
\end{aligned}
$$

where $c_{2 p}^{1}, c_{2 q}^{2}, u_{s}$ are generators of degrees $4 p, 4 q, 2 t-1$ respectively, and

$$
d c_{2 p}^{1}=d c_{2 q}^{2}=0, \quad d u_{2 s-1}=0, \quad d u_{2 s}=c_{2 s}^{2}-c_{2 s}^{1} .
$$

Define $\rho: W L_{\mathfrak{n}} \rightarrow \wedge M$ by

$$
\begin{aligned}
& \rho\left(c_{2 p}^{2}\right)=\Delta\left(\theta_{1}\right) c_{2 p}, \rho\left(c_{2 q}^{2}\right)=\Delta\left(\theta_{2}\right) c_{2 q}, \\
& \rho\left(u_{s}\right)=\Delta\left(\theta_{1}, \theta_{2}\right) c_{s},
\end{aligned}
$$

where $c$ are the Chern polynomials and $\theta_{a}$ are $L_{a}$-orthogonal unitary connections $(a=1,2)$. $\rho$ induces $\rho^{*}: H^{*}\left(W L_{n}\right) \rightarrow H^{*}(M, R)$ and the classes of $i m \rho^{*}$ 
which are not primary characteristic classes are the secondary characteristic classes of $\left(E, L_{1}, L_{2}\right)$.

A computation similar to one in [L1] (see [V3]) gives

$$
\begin{aligned}
& H^{*}\left(W L_{n}\right)=\hat{W} L_{n}=\mathbf{R}\left[c_{2}^{1}, c_{4}^{1}, \ldots, c_{2[n / 2]}^{1}\right] \otimes \\
& \otimes \mathbf{R}\left[c_{2}^{2}, c_{4}^{2}, \ldots, c_{2[n / 2]}^{2}\right] \otimes \wedge\left(u_{1}, u_{3}, \ldots, u_{2[n / 2]+(-1)^{n-1}}\right),
\end{aligned}
$$

and the conclusion is that the secondary characteristic classes are algebraic combinations of the Pontrijagin classes of $L_{1}, L_{2}$ and of the Maslov classes (3.10) [V3].

Therefore, only the Maslov classes are of interest. One has

Theorem 3.2. The Maslov classes $\mu_{h}\left(E, L_{1}, L_{2}\right)$ do not depend on the choice of the $L_{a}$-orthogonal unitary connecitions $\theta_{a}(a=0,1)$, as well as on the choice of the unitary reduction of the structure group of $E$ (i.e., on $J$ ). They only depend on the homotopy classes (via Lagrangian subbundles) of $L_{2}, L_{2}$, and vanish if $L_{1}, L_{2}$ are homotopic to transversal Lagrangian subbundles.

Proof: Except for the last assertion, the results follows by rather clear homotopy arguments. If $L_{1}, L_{2}$ are transversal we may choose $J$ such that $L_{2}=J L_{1}$, and then use $\theta_{1}=\theta_{2}$. By (3.11), we shall obtain $\mu_{2 h-1}=0$

We refer the reader to [V3] for concrete computations of Masiov classes, and we shall finish here by indicating another interesting feature namely, the residual character of the Maslov classes.

Let us first generalize the definition of residues as given in [BB]. Let $M^{m}$. be an oriented differentiable manifold, and $S$ a compact subset of $M$ whose connected components $S_{k}(h=1, \ldots, p)$ have disjoint tubular neighbourhoods $U_{h}$ with projections $\pi_{h}: U_{h} \rightarrow S_{h}$, and $U=U_{h=1}^{p} U_{h}$. Then, a cohomology class $u \in H^{k}(M, \mathbf{R})$ is said to be $S$-residual if for every $U$ as above there are canonically (but not necessarily uniquely) defined $k$-forms $\lambda_{U}$ with a compact support included in $U$ which represent $u$, and such that if $U^{t} \subseteq U, \lambda_{U^{\prime}}^{\prime}=$ $=\lambda_{U}+d \mu$, where supp $\mu=\operatorname{compact} \subset U$.

If this happens, and if $\lambda_{h}=\lambda_{U} / U_{n}$, Poincare duality provides us with a class $\alpha_{h} \in H_{m-k}\left(U_{h}, \mathrm{R}\right)$ characterized by

$$
\int_{\alpha_{h}} \varphi=\int_{U_{h}} \lambda_{h} \wedge \varphi \quad\left(\forall \varphi \in \wedge^{m-k} U_{h}, d \varphi=0\right),
$$

and the homology class

$$
\text { res } S_{h} u=\left(\pi_{h}\right)_{*} \alpha_{h} \in H_{m-k}\left(S_{h}, \mathbf{R}\right),
$$

is called the residue of $u$ along $S_{h}$. By the last condition in the definition of $S$-residuality, the residues do not depend on the choice of $U$. Furthermore, if $M$ is compact it follows obviously that the following residues theorem holds

$$
u=\sum_{h=1}^{p} \mathcal{P}_{M}\left(i_{M}^{h}\right)_{*} \operatorname{res}_{S_{h}} u,
$$


where $\mathcal{P}_{M}$ denotes Poincaré duality on $M$, and $i_{M}^{h}$ is the inclusion $S_{h} \subset M$.

The simplest situation is when $S_{h}$ are submanifolds. For residue theories where $S$ is a stratified set see the recent work of D. Lehmann [L2]. (See also [G1], [G2].) At our knowledge the residual classes studied until now were primary characteristic classes (e.g., Chern classes [BB]). We can prove that the Masiov classes, which are secondary characteristic classes, also have an $S$-residual character along the nontransversality set $S$ of the Lagrangian subbundles $L_{1}, L_{2}$. Therefore, by the residues theorem (3.16) these classes are determined by local information around $S$, at least if $S$ is nice enough as above.

To show the residual character of $\mu_{h}\left(E, L_{1}, L_{2}\right)$, we start with a unitary reduction associated to a complex structure $\tilde{J}$ on $E / M \backslash S$ such that $L_{2}=\tilde{J} L_{1} . \tilde{J}$ exists because $L_{1}, L_{2}$ are transversal along $M \backslash S$. Then, we construct a unitary reduction of $E / M$ by a complex structure $\bar{J}$ which equals $\tilde{J}$ outside a tubulas neighbourhood $V$ such that $\bar{V}=$ compact $\subset U$ (the $U$ of the residuality definition). ( $\bar{J}$ may be obtained by a convenient partition of unity argument, see details in [V5]). Furthermore, we take $\theta_{1}$ to be a $L_{1}$-orthogonal $\bar{J}$. unitary connection. Over $M \backslash S, \theta_{1}$ is also $L_{2}$-orthogonal, and we can construct an

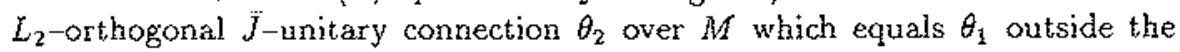
neighbourhood $V$ introduced above. (Use again the partition of unity argument.) Then, by (3.11) the form $\Delta\left(\theta_{1}, \theta_{2}\right) c_{2 h-1}$ has a compact support included in $U$, and it yields the form $\lambda_{U}$ of the definition of $S$-residuality. If $U^{\prime} \subseteq U$, a technical analysis [V5] shows that $\Delta\left(\theta_{0}, \theta_{1}\right) c_{2 h-1}$ is "homotopically deformed" to $\triangle\left(\theta_{0}^{\prime}, \theta_{2}^{\prime}\right) c_{2 h-1}$ via forms with compact support in $U$.

This justifies the residual character of the Maslov classes. 


\section{References}

[AG] V.I. ARnold, A.B. Givental, Symplectic Geometry, Dynamical Systems-4, Itogy Nauki i Tehniki (1985), 7-139, Viniti, Moskwa.

[BB] P. BAUM, R. BOTT, Singularities of holomorphic foliations, J. Diff. Geom. 7 (1972), 279-342.

[Bv] A. BEAUVILLE, Variétés kählériennes dont la première classe de Chern est nulle, J. Diff. Geom. 18 (1983), 755-782.

[D] J.J. DuistermaAT, On global action angle coordinates, Comm. Pure Appl. Math. 33 (1980), 687-706.

[DD] P. DAZORD, TH. DELzANT, Le problème général des variables actions-angles, J. Diff. Geom. 26 (1987), 223-251.

[Dz] TH. DELZANT, Variables actions-angles non commutatives et examples d'images convexes de l'application moment, Thèse de Doctorat de l'Univ. Pierre et Marie Curie (1986), Paris.

$[\mathrm{GH}]$ W. GOLDMAN, M.W. HIRSCH, The radiance obstruction and parallel forms on affine manifolds, Transactions American Math. Soc. 286 (1984), 629-649.

[G1] F. GOME2, A residue formula for characteristic classes, Topology 21 (1982), 101-124.

[G2] F. GOMEZ, De Rham homology for networks of manifolds, Israel $J$. Math. 54 (1986), 110-128.

[GS1] V. Gullemin, SH. STERNBERG, Deformation theory of pseudogroup structures, Memoirs AMS 64 (1966), Providence R.I..

[GS2] V. Gullibmin, Sh. STERnberg, Geometric Asymptotics, Math. Surveys, American Math. Soc. 14 (1977), Providence R.I..

[L1] D. LeHManN, Classes caractéristiques et $J$-connexité des espaces de connexions, Ann. Inst. Fourier Grenable 24(3) (1974), 267-306.

[L2] D. LemMann, Intégration sur les variétés stratifiées, C.R.Acad. Sci. Paris Série I, 307 (1988), 603-606.

[LM] P. LiBERMANN ET CH. M. MARLE, Symplectic geometry and analytical mechanics, D. Reidel Publ. Comp. (1987), Dordrecht.

[M1] P. Molino, Propriétés cohomologiques et propriétćs topologiques des feuilletages à connexion transverse projetable, Topology 12 (1973), 317-325.

[M2] P. Molino, Géométrie des polarisations, In: Feuilletages et quantification géométrique, Sém. Sud-Rhodanien de Geom. II (P. Dazord, N. Desolneux-Moulis, eds.). Collection Travaux en Cours, Hermann (1984), 37-54, Paris.

[Vi] I. VAISMAN, Cohomology and differential forms, M. Dekker, Inc. (1973), New York.

[V2] I. VAISMan, Symplectic curvature tensors, Monatshefte für Math. 100 (1985), 299-327. 
[V3] I. VAISMAN, Symplectic Geometry and secondary characteristic classes, Progress in Math. 72 (1987), Birkhäuser-Boston.

[V4] I. VAISMAN, $d_{f}$-Cohomology of Lagrangian Foliations, Monatshefts für Math. 106 (1988), 221-244.

[V5] I. VAISMAN, Residues of Chern and Maslov classes, Preprint (1987).

[W] A. WEINSTEIN, Symplectic manifolds and their Lagrangian submanifolds, Advances in Math. 6 (1971), 329-346.

Department of Mathematics

University of Haifa

ISRAEL

Rebut el 3 de Novembre de 1988 